Cita bibliográfica: Puig-Cabrera, M. y Foronda-Robles, C. (2018). El Turismo y el alivio de la pobreza: un enfoque desde el prisma de los destinos emergentes en el marco de la Agenda 2030. Investigaciones Turísticas (16), pp. 1-22. http://dx.doi.org/10.14198/INTURI2018.16.01

\title{
El turismo y el alivio de la pobreza: un enfoque desde el prisma de los destinos emergentes en el marco de la Agenda 2030
}

\section{Tourism and poverty alleviation: an approach from the perspective of emerging destinations within the framework of the 2030 Agenda}

Miguel Puig-Cabrera iD, Universidad de Sevilla, España. mpuigcabrera@gmail.com Concepción Foronda-Robles ID, Universidad de Sevilla, España. foronda@us.es

\section{RESUMEN}

El turismo es señalado como un sector clave para la consecución de los Objetivos de Desarrollo Sostenible (ODS). Los beneficios que reporta esta actividad en los destinos tradicionales son incuestionables. No obstante, desde el prisma de los destinos emergentes, las garantías no están aseguradas. El propósito principal de este trabajo es analizar la potencialidad del turismo en los destinos emergentes y sus efectos en el desarrollo socioeconómico, de cara a la Agenda 2030. Se han formulado diversas hipótesis para ser contrastadas en 152 destinos emergentes, buscando un acercamiento a la realidad de estos países desde el turismo y su contribución al desarrollo socioeconómico de la población. Se incluyen 43 de los 48 Países Menos Adelantados (PMA), caracterizados por tener el turismo como una de sus principales fuentes de exportación. La metodología utilizada se basa en un análisis cuatridimensional, a partir de variables macroeconómicas que consideran: 1) las condiciones de pobreza y desigualdad del territorio; 2) el nivel de desarrollo turístico; 3 ) el compromiso de las administraciones públicas con el turismo y 4) el acceso directo por parte de la población hacia los beneficios que reporta el turismo en el territorio. El método de contraste seguido para validar las hipótesis iniciales ha sido a través del coeficiente de correlación de Pearson. Los resultados de este trabajo ponen en entredicho si el desarrollo turístico es la causa del desarrollo en un destino, o, por el contrario, el desencadenante de su subdesarrollo.

Palabras clave: Desarrollo; Turismo; Pobreza; Destino Emergente; Agenda 2030.

\section{ABSTRACT}

Tourism constitutes a key sector for the achievement of Sustainable Development Goals within the framework of Agenda 2030. The benefits brought about by this activity in traditional destinations are unquestionable. Nevertheless, there are no guarantees from the perspective of emerging destinations. The purpose of this research is to analyze the potential of tourism in 
emerging destinations and its effects on socioeconomic development within the framework of the 2017 International Year of Sustainable Tourism for Development and the Agenda 2030. To do this, several hypotheses have been formulated to be tested in 152 emerging destinations. These include 43 out of 48 Less Developed Countries (LDCs) that are characterized by having a tourism activity that represents one of their main sources of exports. Pearson's correlation coefficient has been used to validate the hypotheses. The methodology used is based on a four-dimensional model considering: 1) poverty conditions and territorial inequalities; 2 ) the degree of tourism development; 3) the commitment of public institutions to tourism and 4) the direct access of the local population to the benefits brought about by tourism. The findings call into question whether tourism development is the cause of the development of a destination or, on the contrary, contributes to its underdevelopment.

Keywords: Development; Tourism; Poverty; Emerging Destination; Agenda 2030.

\section{INTRODUCCIÓN}

El alivio de la pobreza se ha convertido en uno de los principales ejes estratégicos de los organismos internacionales (Holden et al., 2011), siendo cada vez más numerosas las instituciones sensibilizadas en esta materia, principalmente a escala internacional, como el Banco Mundial, el Fondo Monetario Internacional (FMI), la Organización Mundial de Comercio (OMC), la Organización Mundial del Turismo (OMT) o el Consejo Mundial de Viajes y Turismo (WTTC).

En el año 1970 surgen los primeros movimientos contra la pobreza, plasmados en los Programas de Ajuste Estructural impuestos por el Banco Mundial, junto a los Programas de Estabilización del FMI. La finalidad era acelerar la transferencia de recursos hacia los países menos avanzados para combatir la deuda externa (Figueroa-Hernández et al., 2015).

Ante una situación de ineficacia y falta de resultados, en 1990, intervienen el Banco Mundial y el Programa de las Naciones Unidas para el Desarrollo (PNUD) junto a la OCDE, que establecen los Objetivos Internacionales para el Desarrollo (Collier y Dollar, 2001), y que terminarán convirtiéndose en los Objetivos de Desarrollo del Milenio (ODM). Posteriormente, los Documentos de Estrategia de Lucha contra la Pobreza (FMI, 2012) son una alternativa que vela por una participación conjunta de la población, la iniciativa privada y pública.

Entre 1981 y 2015 , el número de personas que subsiste con menos de $1,90 \$$ al día se reduce de manera considerable, pasando del $44 \%$ de la población mundial al 9,6\%, pese a un aumento de la demografía. Esto se traduce en que 702 millones de personas aún viven en condiciones de pobreza extrema (Banco Mundial, 2016), lo que continúa siendo una cifra inaceptablemente alta. Esto tampoco significa que no haya población que se haya podido empobrecer aún más, si cabe.

Por su parte, ya en el discurso de la Conferencia de las Naciones Unidas sobre Comercio y Desarrollo (ONU, 1964), se resalta la importancia que tiene la actividad turística 
en las economías en vías de desarrollo. El concepto de turismo pro-pobre (PPT) surge en el Departamento para el Desarrollo Internacional de Reino Unido (DFID, 1999), como una propuesta para incorporar a los colectivos más desfavorecidos al mercado mediante intervenciones de proyectos turísticos comercialmente viables que incrementasen el empleo y las oportunidades empresariales (Ashley et al., 2000; Harrison, 2008). Esta modalidad pretende beneficios netos para la población a partir de la rentabilidad turística del destino, lo que genera oportunidades para una mejora en sus condiciones de vida, y sitúa a la comunidad local como eje central del proceso de planificación y desarrollo turístico (Idelhadj et al., 2012).

Los artículos científicos lo han conceptualizado como el turismo y el alivio de la pobreza (Scheyvens y Russell, 2012); en otros casos, como la reducción de la pobreza (Spenceley y Meyer, 2012), y otros lo llevan al extremo de la posibilidad de la erradicación de la misma (Casas et al., 2012). Por otro lado, se ha prestado escaso énfasis en el impacto del turismo (Gutiérrez-Pérez et al, 2014). Unos autores defienden los efectos positivos del turismo en el desarrollo económico (Spenceley y Meyer, 2012) e incluso llegan a medir empíricamente dicho impacto (Blake, 2008); otros argumentan, que ello no está suficientemente demostrado (Harrison, 2008; Holden et al., 2011; Zhao y Ritchie, 2007).

En este contexto, la OMT apuesta por la actividad turística para la consecución de los Objetivos de Desarrollo Sostenible (ODS) en la Agenda 2030.

\section{OBJETIVOS}

El propósito principal de este trabajo es analizar la potencialidad de la actividad turística en los destinos emergentes y sus efectos en el desarrollo social y las desigualdades económicas, de cara a la Agenda 2030, y diseñar relaciones entre indicadores que midan cómo el turismo contribuye a la calidad de vida de la población.

La innovación del artículo es establecer la realidad de estos países; las relaciones de los servicios turísticos con el propio hecho social, para así dar lugar a nuevos planteamientos científicos, que aporten mayor calidad de vida en los destinos emergentes.

\section{MARCO TEÓRICO}

\subsection{Teorías de turismo y desarrollo}

De manera tradicional, se conocen cuatro posturas o corrientes ideológicas (liberalismo, criticismo, alternativismo y post-estructuralismo) que relacionan el desarrollo turístico como un motor capaz de contribuir al desarrollo socioeconómico de la población (tabla 1.1). Asimismo, existen otras (teoría de la modernización, la dependencia y el desarrollo sostenible), que comparten rasgos con algunas de las posturas tradicionales. 
Tabla 1. Teorías de turismo y desarrollo

\begin{tabular}{|c|c|}
\hline Teorías & Rasgos esenciales \\
\hline $\begin{array}{l}\text { Liberalismo } \\
(1950-)\end{array}$ & $\begin{array}{l}\text { - Uso intensivo de recursos productivos para maximizar la generación de divisas. } \\
\text { - Turismo de masas=desarrollo local. }\end{array}$ \\
\hline $\begin{array}{l}\text { Teoría de la } \\
\text { Modernización } \\
\text { (1950-1960) }\end{array}$ & $\begin{array}{l}\text { - Desarrollo económico como proceso endógeno. } \\
\text { - Reducción gradual desigualdades económicas (“impulsos económicos" y "polos } \\
\text { de crecimiento"). } \\
\text { - Uso intensivo de recursos en el camino hacia la modernización. } \\
\text { - Indicadores macroeconómicos como herramientas para evaluar el proceso de } \\
\text { desarrollo. }\end{array}$ \\
\hline $\begin{array}{l}\text { Criticismo } \\
(1960-)\end{array}$ & $\begin{array}{l}\text { - Desarrollo turístico sinónimo de desigualdad y pobreza. } \\
\text { - Efectos nocivos sobre las condiciones de vida de la población y sobre el } \\
\text { territorio. }\end{array}$ \\
\hline $\begin{array}{l}\text { Teoría de la Dependencia } \\
\qquad(1960-1970)\end{array}$ & $\begin{array}{l}\text { - Países desarrollados crecen a costa de explotar los recursos de los países en } \\
\text { vías de desarrollo. } \\
\text { - Cuanto mayor desarrollo en los países capitalistas, mayores índices de } \\
\text { subdesarrollo en los países pobres. } \\
\text { - El desarrollo turístico conlleva a la desigualdad si el control de la actividad está } \\
\text { en manos de la inversión extranjera o las clases más altas. } \\
\text { - El desarrollo turístico sólo puede ser efectivo si es gestionado por la } \\
\text { comunidad local. }\end{array}$ \\
\hline $\begin{array}{l}\text { Alternativismo } \\
(1980-)\end{array}$ & $\begin{array}{l}\text { - Primer concepto de sostenibilidad. } \\
\text { - Desarrollo turístico atendiendo al pilar económico, social y medioambiental. } \\
\text { - Equilibrio de los tres pilares sinónimo de desarrollo local. }\end{array}$ \\
\hline $\begin{array}{l}\text { Post-estructuralismo } \\
\text { (1990-) }\end{array}$ & $\begin{array}{l}\text { - Turismo como nueva disciplina científica. } \\
\text { - Turismo como fenómeno complejo para su comprensión en el alivio de la } \\
\text { pobreza. } \\
\text { - Numerosos y diversos puntos de vista para su comprensión (relativismo). }\end{array}$ \\
\hline $\begin{array}{l}\text { Desarrollo sostenible } \\
\qquad(1990-)\end{array}$ & $\begin{array}{l}\text { - Turismo sostenible como vía para el desarrollo. } \\
\text { - Concepto de sostenibilidad ampliado. } \\
\text { - Orientado a destinos en vías de desarrollo y a destinos desarrollados. }\end{array}$ \\
\hline
\end{tabular}

Elaboración propia basada en Harrison, 2015 y Sharpley y Telfer, 2014.

\subsubsection{Liberalismo}

En la década de los años 1950, se populariza el liberalismo, que aprovecha el evidente crecimiento de la actividad turística en el marco internacional para generar beneficios económicos de forma masiva para la población involucrada (Jafari, 2005).

Las administraciones públicas destinan todos los medios necesarios para que el turismo sea explotado, centrando sus esfuerzos en esta actividad, que pasa a ser concebida como una "panacea" (Sharpley y Telfer, 2014). En este sentido, estos destinos tienden a una economía de "monocultivo" como fuente de ingresos prioritaria (Harrison, 2015). En la historia del turismo, el liberalismo se corresponde con lo que se conoce como el "fordismo", basado en una oferta turística masiva de escasa especialización y hacia una demanda turística aún más masiva.

De esta manera, el turismo se entiende como sinónimo de desarrollo local mediante la creación de empleo, generación de divisas, uso de infraestructura y productos locales, mejora de la educación, fomento del entendimiento internacional, reducción de barreras culturales y/o fortalecimiento del valor patrimonial (García y Lavalle, 2012). 
En este momento, se acude a la actividad turística para la erradicación de la pobreza, originando un escenario donde dichos destinos cuenten con los medios suficientes para ofertar y abastecer a la demanda, dando lugar a una generación de beneficios.

\subsubsection{Modernización}

En las décadas de 1950 y 1960 cobra intensidad la teoría de la modernización, coexistiendo con la teoría liberalista. Este enfoque sostiene que el desarrollo económico es impulsado a partir de un proceso endógeno que tiene lugar desde dentro de la propia economía por las autoridades competentes (Sharpley, 2009). Este proceso va desarrollándose de manera progresiva a partir de diversas etapas (Rostow, 1960) que llevan a la "modernización", una situación caracterizada por el uso intensivo de los recursos productivos para abastecer al consumismo de masas. Las fases por las que pasa la economía son resultado de los continuos "impulsos económicos" o "polos de crecimiento" (Browett, 1985; Perroux, 1955), generando beneficios adicionales que revierten directamente a los grupos más desfavorecidos y, por ende, conllevan la reducción de las desigualdades económicas (Opperman, 1993).

En este sentido, la teoría de la modernización señala la actividad turística como un impulso económico con el suficiente potencial para reducir las desigualdades (Sharpley, 2009), apoyándose en una serie de indicadores macroeconómicos como el PIB, exportaciones turísticas o el ingreso per cápita (Fitzgerald, 1981). Esta teoría apuesta por una política proteccionista y un turismo de masas para reducir las desigualdades económicas en el destino.

\subsubsection{Criticismo}

El criticismo cobra importancia en los años 1960, subrayando que el desarrollo turístico masivo por el que estaban pasando muchos destinos conlleva una considerable degradación de los mismos, a partir de una serie de efectos adversos (Jafari, 2005). En este sentido, el turismo se concibe como una de las causas directas de la existencia de situaciones de desigualdad y pobreza, deteriorando las condiciones de vida de la población, y degradando su entorno (Korstanje, 2015). Partiendo de las dos teorías anteriores, el hecho que el turismo sea considerado como una panacea, lleva a las administraciones a desinvertir fondos públicos destinados a los residentes para reinvertirlos en servicios que satisfagan las necesidades de los turistas, buscando el crecimiento del número de visitas anuales al destino. De esta manera, ante la falta de compromiso pro-pobre, la inversión pública se enfoca más en la construcción de un aeropuerto o la pavimentación de una carretera, que en la capacitación de la comunidad para su inserción en el sector turístico.

Comienzan a identificarse algunos efectos adversos asociados al turismo de índole económica (dependencia del exterior, desigualdad económica, inflación, riesgo de exclusión social...), sociocultural (alteración comportamiento población residente, choques culturales, mercantilización de la cultura...) y ambiental (superación capacidad de carga, degradación física monumentos y los espacios naturales, generación de residuos...) (Archer et al., 2005). 


\subsubsection{Dependencia}

Esta teoría del desarrollo bebe de los ideales marxistas, defendiendo que el desarrollo del capitalismo de los destinos desarrollados perpetúa las condiciones de subdesarrollo en que se encuentran los destinos con menos recursos (Dos Santos, 1970), en la medida en que se empoderan de sus recursos para explotarlos y seguir creciendo a su costa. En este sentido, el desarrollo turístico es inherente a la creación de mayores desigualdades económicas cuando éste se sustenta en el sistema capitalista (Blázquez-Salom, 2012).

Esta teoría apunta que la cadena de valor económica está configurada en torno a los grandes núcleos económicos (destinos desarrollados), que se alimentan de los núcleos periféricos (destinos en vías de desarrollo) para seguir creciendo (Harrison, 2015). Así, este turismo podría concebirse como una vía de dominación y control, pudiendo asemejarse a un nuevo intento de "colonización" (Mowforth y Munt, 1998).

En relación a la actividad turística, esta postura señala que los intereses de la inversión extranjera y de las clases dominantes son los únicos que se satisfacen cuando algunos de estos agentes tienen acceso a su control (Britton, 1982). De esta manera, se apuesta por una actividad turística gestionada por la comunidad (Phillips, 1977). Para ello, esta teoría apuesta por implantar políticas socialistas y dejar a un lado los ideales capitalistas (Sharpley, 2009).

\subsubsection{Alternativismo}

En 1980, el alternativismo busca el equilibrio entre el binomio beneficio-coste para el desarrollo turístico (Muñoz, 2005). Comienza a concebirse una nueva forma de interacción entre los destinos emergentes y los destinos desarrollados, en la medida en que el proceso debe ser impulsado y controlado desde el propio destino (Sharpley, 2009). Así, el concepto de sostenibilidad comienza a integrarse (Butler, 1999; Jafari, 2005). Las políticas turísticas dejan de tener un contenido puramente económico, para incluir también aspectos sociales y medioambientales, relacionados con las condiciones de vida de la comunidad y la protección de su patrimonio natural y/o cultural (Redclift, 1987).

Este enfoque respalda las formas de turismo que se basan en el respeto a la comunidad anfitriona, el empleo de recursos locales de una manera responsable (Muñoz, 2005). Partiendo de una concepción más positiva, comienzan a popularizarse las iniciativas turísticas locales que pretenden beneficiar a los más desfavorecidos, minimizar los daños medioambientales y buscar una buena relación entre población local y visitantes.

Actualmente, el turismo sostenible continúa siendo una apuesta en el mercado turístico, especialmente en destinos emergentes, que acuden a estas modalidades para asegurar un desarrollo socioeconómico equitativo y sostenible.

\subsubsection{Post-estructuralismo}

Por su parte, en los años 1990, el post-estructuralismo planteó el paradigma del desarrollo turístico y el alivio de la pobreza desde el campo científico, convirtiéndose así en una nueva materia de estudio (Jafari, 2005). 
En este sentido, el turismo es abordado desde un punto de vista holístico, analizando su estructura, sus funciones, así como las interacciones y los resultados en el destino. Esta corriente deja a un lado los impactos del turismo para pasar a concebirlo como un fenómeno que se refleja en el destino (Davis, 2001). La interacción que tienen los diversos actores en la cadena de valor del destino, da lugar a una concepción propia que contrastará en mayor o menor medida con la del resto de actores, dando un significado distinto al espacio turístico.

La combinación de estos puntos de vista (Wolf, 1982) puede ser utilizada para el análisis y la interpretación del turismo en un destino. Asimismo, el post-estructuralismo señala a la población local como un factor clave, en la medida en que puede resistir, destruir, manipular o transformar el turismo para su propio beneficio (Jafari, 2005).

\subsubsection{Desarrollo Sostenible}

El enfoque del desarrollo sostenible surge de la conjunción de diversos pensamientos: por un lado, las teorías más a favor de esta actividad, que ya se han indicado con anterioridad, y por otro, el enfoque de la sostenibilidad (Sharpley, 2009). De esta combinación, el turismo es un fenómeno con importante potencial para contribuir al desarrollo socioeconómico de la población.

En la actualidad, tras los Objetivos de Desarrollo del Milenio (ODM) y la delimitación de los ODS en 2015, y partiendo de un mayor nivel de profundización de este fenómeno en el campo de la ciencia, se apuesta por un turismo sostenible más allá del pilar económico, social y ambiental, y se orienta no solo hacia los destinos en vías de desarrollo, sino también hacia los desarrollados (Ariza, 2016; Hardy \& Pearson, 2016).

Así, se señalan directamente los tres pilares tradicionales de la sostenibilidad en un sentido más amplio, enumerando directamente aspectos como el fin de la pobreza, la educación, la igualdad de género, la salud, la protección de los recursos naturales, las energías renovables y/o la paz a nivel mundial (OMT, 2016).

En definitiva, los enfoques teóricos que relacionan el turismo y el alivio de la pobreza, sea desde una postura positiva o negativa hacia esta actividad, abordan cuatro elementos comunes que son clave: 1 ) la incidencia de la actividad turística sobre la economía del destino, y, en particular, sobre las condiciones de pobreza y desigualdad existentes; 2 ) el nivel de desarrollo turístico actual versus su potencialidad, como oportunidad de riqueza de cara al futuro; 3) el modelo de gobernanza turística por parte de las autoridades competentes, reflejando el respaldo de la iniciativa pública hacia esta actividad; y 4) el acceso directo por parte de la población hacia los beneficios que reporta el turismo en el territorio. Son estas mismas cuatro dimensiones sobre las que se sustenta el presente trabajo para analizar la relación entre turismo y pobreza en los destinos emergentes (véase apartado IV).

\subsection{Los objetivos de desarrollo sostenible y la Agenda 2030}

Partiendo de las lecciones aprendidas del turismo durante los Objetivos del Milenio (ODM), y en el marco de la Agenda 2030, la OMT (2015) señala el turismo como uno de los 
principales campos de acción para el cumplimiento de los ODS, vinculándolo de manera directa sobre tres de estas metas: promover el crecimiento económico inclusivo y sostenido (ODS 8); garantizar modalidades de consumo y producción sostenibles (ODS 12) y conservar y utilizar de forma sostenible los recursos marinos (ODS 14). Esto se materializa en la creación de planes y programas, señalando al turismo de manera explícita como uno de los sectores de intervención.

Asimismo, el turismo es relacionado de manera indirecta con la consecución de los 14 ODS restantes (Puig-Cabrera \& Foronda Robles, 2017), siendo considerado un sector con potencialidad para incidir sobre la vertiente socioeconómica de la población: poner fin a la pobreza y el hambre en el mundo (ODS 1 y 2); mejorar la salud y el bienestar de la población (ODS 3), cubrir las carencias educativas (ODS 4), y fomentar la igualdad de género (ODS 5).

Asimismo, en la I Conferencia Mundial sobre Turismo para el Desarrollo (OMT, 2016) se puntualiza la necesidad de investigar en el campo del turismo pro-pobre para facilitar la medición de sus efectos sobre el índice de pobreza de los destinos emergentes. Esto vuelve a convertir al turismo en uno de los focos de atención para lograr los Objetivos de Desarrollo Sostenible, de cara a la Agenda 2030.

\section{METOdOLOGÍA}

\section{1. Ámbito de estudio}

Los países objeto de estudio de esta investigación lo conforman las 152 economías emergentes consideradas a nivel internacional, atendiendo a las publicaciones periódicas del FMI (2016) y la OMT (2017a): World Economic Outlook y World Tourism Highlights. Estos destinos se caracterizan por compartir dos rasgos comunes: por un lado, más del $50 \%$ del total de sus ingresos por exportación deriva de combustibles $u$ otros productos primarios. Por otro lado, su Posición de Inversión Internacional (PII) es igual o inferior a 0, lo que supone que estos países cuenten con un mayor número de inversiones extranjeras (pasivo exterior) que propias en otros países (activo exterior). En este grupo figuran también 43 de los 48 Países Menos Adelantados (PMA) designados en la Lista de los PMA (ONU, 2016b), focos prioritarios a la hora de elaborar nuevas actuaciones en materia de desarrollo local y alivio de la pobreza, y caracterizados por tener al turismo como uno de los principales motores de exportación en su economía (ITC, 2011). Cualquiera que sea el nivel de desarrollo turístico de uno de estos destinos, difícilmente se pueda afirmar que esta actividad sea inexistente. En este sentido, este trabajo utiliza indistintamente los términos "destino", "economía" o "país", considerando que la muestra conformada permite obtener una visión global para conocer cómo el volumen de turismo de estos países se relaciona con sus condiciones de pobreza.

\subsection{Materiales y fuentes}

Partiendo del supuesto que el turismo se encuentra vinculado a la reducción de la pobreza en un destino (Croes y Venegas, 2008; OMT, 2017b), se han elaborado hipótesis 
iniciales planteando la existencia de correlación entre variables macroeconómicas, buscando incidir en la relación entre el turismo y su potencialidad para el desarrollo socioeconómico dentro de los destinos, a partir de un análisis con datos de sección cruzada multipaís. Las fuentes estadísticas empleadas son: el Banco Mundial (2016), que utiliza datos de procedencia mixto, combinando los de otras fuentes oficiales, con los propios recopilados por este organismo. Esta fuente se rige por el Sistema de Cuentas Nacionales de la ONU (2008), aplicando una metodología normalizada, que asegura la fiabilidad de los datos en cuanto a terminología, periodo y unidad de medida. Por su parte, se ha acudido al Consejo Mundial de Turismo y Viajes (WTTC, 2016), que sigue los principios metodológicos sugeridos por la ONU (2011) para la elaboración de las Cuentas Satélites de Turismo, ofreciendo datos históricos, así como previsiones futuras para un horizonte temporal de diez años.

Ambas fuentes son utilizadas con frecuencia por la comunidad académica, sea para realizar un tratamiento estadístico de manera directa con estos datos (Hanafiah et al., 2016; Simas et al., 2017), o como información complementaria al análisis que realizan (Arshad et al., 2018; Koens \& Wood, 2017). Por tanto, estas fuentes han sido escogidas atendiendo tanto a la consistencia de los datos, como a la normalización en la metodología de recopilación y almacenamiento.

El horizonte temporal fijado es el año 2014, al ser este último del que se disponen los datos más recientes para la mayor parte de los indicadores utilizados. Éstos han sido insertados en una base de datos (Excel 15.0) y se ha utilizado el análisis estadístico a través de SPSS 22.0 para contrastar y comprobar las hipótesis inicialmente planteadas por medio del coeficiente de correlación de Pearson. De esta manera, se han identificado las variables con relaciones significativas entre la actividad turística y su potencialidad para reducir las brechas económicas y las condiciones de pobreza en el destino.

Se ha elaborado cartografía coroplética para representar las variables analizadas y con correlaciones significativas a partir de ArcGIS 10.4.1. Esta herramienta permite representar de manera gráfica la distribución e intensidad de las variables analizadas sobre el territorio.

\subsection{Indicadores}

Los indicadores utilizados en esta investigación (tabla 3) abordan cuatro dimensiones: 1) las condiciones de desigualdad y pobreza existentes en el destino y su materialización en el territorio; 2) el nivel de desarrollo turístico; 3) el compromiso de las administraciones públicas y 4) el acceso directo por parte de la población hacia los beneficios que reporta el turismo en el territorio. dimensiones sirven para clasificar los indicadores en un grupo u otro en función de lo que señalan: 
Tabla 3. Indicadores de turismo y pobreza

\begin{tabular}{|c|c|c|c|}
\hline Dimensión & Indicador & Descripción & Referencias \\
\hline \multirow{3}{*}{$\begin{array}{l}\text { Pobreza y } \\
\text { desigualdad }\end{array}$} & $\begin{array}{l}\text { Índice de pobreza } \\
\text { (IP) }\end{array}$ & $\begin{array}{l}\text { Porcentaje de población con ingresos } \\
<1,90 \$ / \text { día }\end{array}$ & $\begin{array}{l}\text { Banco Mundial, 2016; } \\
\text { CEPAL, } 2012\end{array}$ \\
\hline & $\begin{array}{l}\text { Participación } \\
\text { ingresos } \\
20 \% \text { menos } \\
\text { remunerado } \\
\text { (PI20) }\end{array}$ & $\begin{array}{l}\text { Porcentaje de personas con menores } \\
\text { ingresos en el destino }\end{array}$ & $\begin{array}{l}\text { Banco Mundial 2016; } \\
\text { Blake, } 2008\end{array}$ \\
\hline & $\begin{array}{l}\text { Población } \\
\text { residente en } \\
\text { slums (Slums) }\end{array}$ & $\begin{array}{l}\text { Porcentaje población residente en } \\
\text { viviendas que carecen de acceso a } \\
\text { agua potable; servicios comunitarios; } \\
\text { superficie habitable; aseo y/o materiales } \\
\text { de construcción estables }\end{array}$ & $\begin{array}{l}\text { Banco Mundial, 2016; } \\
\text { Frenzel et al., 2012; } \\
\text { ONU, } 2003\end{array}$ \\
\hline $\begin{array}{l}\text { Actividad turística } \\
\text { en destino }\end{array}$ & $\begin{array}{l}\text { PIB turístico } \\
\text { agregado (PIBt) }\end{array}$ & $\begin{array}{l}\text { Contribución total del turismo a la } \\
\text { economía }\end{array}$ & $\begin{array}{l}\text { Puppim, 2003; WTTC, } \\
2016 .\end{array}$ \\
\hline $\begin{array}{c}\text { Compromiso } \\
\text { administraciones } \\
\text { públicas }\end{array}$ & $\begin{array}{c}\text { Gasto público en } \\
\text { actividad turística } \\
\text { (GPT) }\end{array}$ & Inversión pública en el sector turístico & $\begin{array}{l}\text { Webster y Ivanov, } \\
\text { 2014; WTTC, } 2016\end{array}$ \\
\hline $\begin{array}{l}\text { Efectos directos del } \\
\text { turismo }\end{array}$ & $\begin{array}{l}\text { Tasa de retorno } \\
\text { (TR) }\end{array}$ & $\begin{array}{l}\text { Porcentaje de ingresos turísticos que } \\
\text { revierten a otras economías }\end{array}$ & $\begin{array}{l}\text { Baretje, 1982; Britton, } \\
\text { 1982; Lejárraga y } \\
\text { Walkenhorst, 2007; } \\
\text { Sandbrook, } 2010 .\end{array}$ \\
\hline
\end{tabular}

Elaboración propia basada en Banco Mundial (2016) y WTTC (2016).

- Atendiendo a las condiciones de pobreza y desigualdad, el IP es una variable clásica en el análisis de la población que vive por debajo del umbral de pobreza fijado. De manera complementaria, se ha incluido PI20 para indagar sobre posibles relaciones entre los estratos de la población con menos ingresos y alguna de las variables del resto de dimensiones. Asimismo, se acude a Slums como variable testigo que plasma la materialización de la pobreza en el territorio. Todo ello buscando conocer si esa materialización guarda alguna relación con el nivel de desarrollo turístico, el compromiso público con respecto a esta actividad, o la propensión de los ingresos a quedar retenidos en el destino.

- Para evaluar el nivel de desarrollo turístico en el destino, se ha seleccionado PIBt, que engloba la contribución directa e indirecta de esta actividad a la economía. Por tanto, esta variable permite identificar potenciales relaciones entre el volumen de turismo actual de un país y sus condiciones de pobreza y desigualdad, así como la vinculación de esta variable a la dotación de recursos públicos.

- Se ha optado por GPT como reflejo del compromiso de las autoridades competentes. En este sentido, debe tenerse en cuenta que la dotación de recursos públicos en el turismo puede tener diversos fines: creación de infraestructura, reforma del marco legal, incentivos al emprendimiento, así como otros planes y programas. Es relevante conocer si, por ejemplo, existe vinculación entre estas partidas de gastos y alguna de las variables vinculadas a las condiciones de pobreza.

- Para conocer cómo se traduce el modelo turístico actual del destino en beneficios económicos para la población, se ha acudido a la TR, puesto que refleja la propensión de 
los ingresos turísticos a ser retenidos por la economía, frente a la posibilidad de revertir a otras economías.

\subsection{Hipótesis}

Para estudiar las relaciones entre los indicadores, se han formulado hipótesis iniciales sugiriendo la existencia de una correlación entre dos variables. Para la comprobación de estas hipótesis, se ha acudido al análisis de coeficiente de correlación de Pearson (Lee, 2013; Webster y Ivanov, 2014) entre las distintas variables cuantitativas, valorando su direccionalidad, su fuerza e intensidad.

Se recogen a continuación las hipótesis iniciales contempladas, deduciendo que, en caso de no ser válidas, se aceptará la correspondiente hipótesis alternativa, es decir, la inexistencia de correlación entre las dos variables $(r=0)$ :

Tabla 4. Matriz de correlación de Pearson entre indicadores pro-pobre

\begin{tabular}{|c|c|c|c|c|c|c|}
\hline & IP & PI20 & Slums & PIBt & GPT & TR \\
\hline IP & 1 &,- 279 &, 164 &, 059 &,- 072 &, 071 \\
\hline PI20 &,- 279 & 1 &, 039 &, 010 &, 056 &, 113 \\
\hline Slums &, 164 &, 039 & 1 & $-0,329 * *$ &,$- 323^{* *}$ &, 018 \\
\hline PIBt &, 059 &, 010 &,$- 329 * *$ & 1 &, $785^{* *}$ &, 010 \\
\hline GPT &,- 072 &, 056 &,$- 323^{* *}$ &, $785^{* *}$ & 1 &, 030 \\
\hline TR &, 071 &, 113 &, 018 &, 010 &, 030 & 1 \\
\hline
\end{tabular}

**. La correlación es significativa en el nivel 0,01 (bilateral).

Elaboración propia

Tras el tratamiento estadístico, y atendiendo a los coeficientes de correlación de Pearson (tabla 4), se aceptan tres de las hipótesis iniciales, significativas al 1\%:

- H1: La contribución de la actividad turística del destino a su economía (PIBt) guarda relación con el índice de personas que reside en Slums $(r=-0,329)$.

- H2: El gasto público en turismo (GPT) se encuentra ligado a la contribución de este sector a la economía del destino (PIBt) $(r=0,785)$.

- H3: El gasto público en turismo (GPT) está correlacionado con el índice de personas que reside en Slums $(r=-0,323)$.

Para el caso de las hipótesis restantes, se rechazan las correlaciones ( $\mathrm{H} 4-\mathrm{H} 10)$, aceptando las correspondientes hipótesis alternativas. Descartar las hipótesis iniciales no implica que no exista relación.

\subsection{Limitaciones}

Un análisis estadístico correlacional permite conocer si existe relación significativa entre variables pudiendo tratarse de dos tipos: 1 ) directa, cuando ambas variables 
crecen o decrecen en el mismo sentido o; 2) inversa, cuando estas variables toman sentido contrario.

La limitación de esta metodología es que no permite abordar la causalidad de las variables, lo que implica que, a pesar de conocer la existencia de una relación significativa, no es posible realizar interpretaciones firmes que expliquen su causa.

Desde el punto de vista territorial y turístico, los análisis estadísticos de correlación son de gran utilidad para conocer, a priori, si la concentración de una variable en el territorio guarda relación con el resto. El análisis ha permitido conocer si existe relación significativa, así como su naturaleza (directa o inversa). En definitiva, las conclusiones obtenidas deben ser consideradas con la precaución de no poderse establecer la relación causa-efecto.

\section{RESULTADOS}

Las tres relaciones $(\mathrm{H} 1, \mathrm{H} 2$ y $\mathrm{H} 3)$ se plasman distintas según la región y los patrones de comportamiento. Se han observado variables latentes que revelan aspectos significativos en el análisis, pese a la falta de correlación.

\section{H1. La contribución de la actividad turística del destino a su economía guarda relación con el índice de personas que reside en Slums}

La relación entre el PIBt y Slums es inversa (tabla 3). El hecho de que ambas variables tengan correlación negativa, sugiere que el desarrollo turístico podría representar una oportunidad para la reducción de los Slums.

Figura 2. Distribución del PIBt y Slums

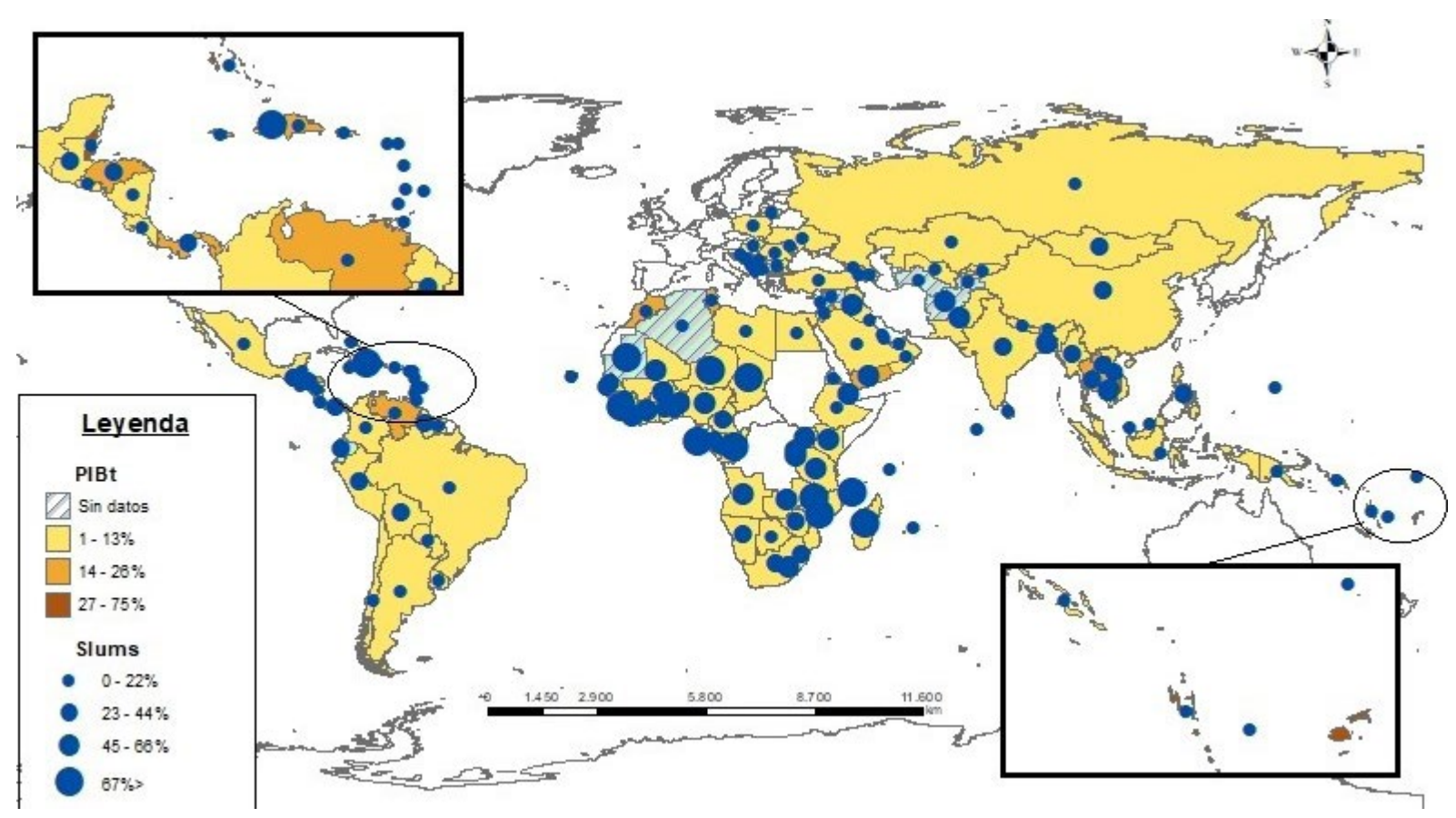

Elaboración propia basada en WTTC (2016) y Banco Mundial (2016c). 
En la figura 2, se observa que el continente africano tiende a concentrar mayores índices de Slums y menores de PIBt, con respecto a América Latina o Asia. Destacan los casos de Mali, Sierra Leona, Guinea, Liberia, y Costa de Marfil. Por el contrario, América Latina tiende a concentrar mayores niveles de PIBt y menores de Slums, como son los casos de Belice, Costa Rica y Honduras. Asia se encontraría en una posición intermedia, existiendo destinos que tienden más a las condiciones de África (Bután o Brunei Darussalam), así como otros a las de América Latina (Laos o Camboya).

Si los beneficios que genera el desarrollo turístico en estos destinos son verdaderamente accesibles para la población más desfavorecida, la proporción de Slums podría disminuir. En caso de no ser accesibles, todo apuntaría que los beneficios serían acaparados por las "élites de poder" (Bonometti y Ruiz, 2010; Hall, 2010) o la inversión extranjera, por tanto, se estarían generando mayores desigualdades económicas en el destino, y el índice de Slums sería mayor.

Haciendo referencia a la condición clásica de Pigou-Dalton (Dalton, 1920; Pigou, 1912), un país tiende al bienestar económico a medida que la riqueza se redistribuye a través de la transferencia de recursos desde los estratos con más ingresos, hacia los colectivos más desfavorecidos, desembocando en una reducción de la desigualdad socioeconómica en el territorio.

En este sentido, el turismo se podría convertir en una actividad con potencialidad para redistribuir la renta y mejorar la calidad de vida de la población más desfavorecida, a partir de la creación de nuevos nexos que permiten la incorporación de estos colectivos en la cadena de los diversos sectores en los que se encuentra el turismo presente, sea de manera directa o indirecta.

\section{H2. El gasto público en turismo se encuentra ligado a la contribución de este sector a la economía del destino}

La relación entre el GPT y el PIBt es directa (tabla 2). Atendiendo a la naturaleza de esta relación, entra en juego el grado de control de la actividad turística por parte de las autoridades nacionales frente a la inversión extranjera.

Esta hipótesis demuestra que la iniciativa pública en los destinos emergentes podría tener un papel relevante en todas las fases del proceso de desarrollo turístico, favoreciendo que esta actividad adquiera un mayor peso en la economía del destino, y, por tanto, pudiendo dotar de medios para que la población desfavorecida tenga la oportunidad de acceder a la cadena de valor turística y beneficiarse.

Asimismo, esta relación sugiere que los recursos públicos destinados al sector sean la respuesta "natural" de las administraciones públicas en su lucha por la hegemonía de la actividad turística en el destino para marcar sus propias "reglas del juego", atendiendo a los intereses de la población, de manera prioritaria a las voluntades del resto de actores o del propio turista. En ambos casos, la dotación de recursos públicos se convertiría en una vía para el empoderamiento de las autoridades competentes para incidir sobre el control de la actividad turística. 
Figura 3. Distribución del PIBt y GPT

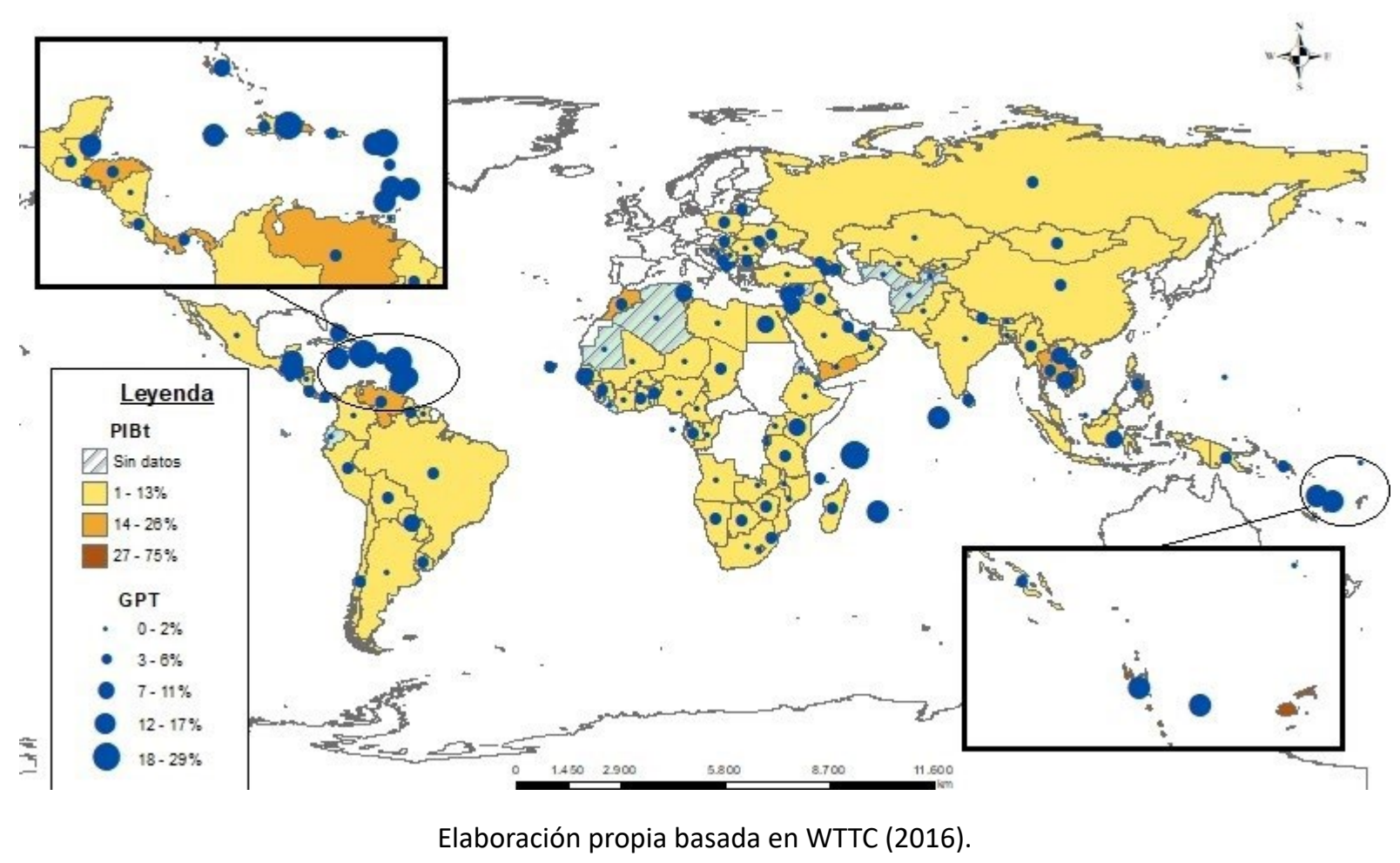

Atendiendo a la figura 3 , se observa que los destinos africanos serían los que concentran menores índices de PIBt y GPT, como son los casos de Níger, Uganda o Etiopía. En la posición contraria, los continentes latinoamericanos presentarían los mayores niveles de PIBt y de GPT. Destacan República Dominicana y Las Bahamas. Al igual que en la hipótesis anterior, los destinos asiáticos tendrían una posición intermedia entre África y América Latina, como Papúa Nueva Guinea y Filipinas, que experimentan condiciones opuestas.

\section{H3. El gasto público en turismo está correlacionado con el índice de personas que reside en Slums}

Se han hallado relaciones significativas entre el GPT y los Slums. El hecho que la correlación entre ambos indicadores sea inversa en estos destinos invita a la reflexión. Independientemente de la causalidad de la relación, la aceptación de esta hipótesis prueba que el gasto público se puede convertir en una herramienta para mejorar las condiciones de vida de la población, a través del turismo. En este sentido, cada unidad monetaria de inversión pública en turismo podría contribuir a una situación de menor desigualdad en el destino.

El compromiso de las autoridades competentes para favorecer que el turismo beneficie a la población, o la capacidad de crear mecanismos efectivos para ello, implica considerar la proporción de los recursos públicos que, en primer lugar, van dirigidos a beneficiar a la población con menos recursos, y, en segundo lugar, consiguen beneficiar a los destinatarios. 
Siguiendo el ejemplo de la condición de Pigou-Dalton, el papel de las administraciones públicas, en este caso, sería, por medio de la inversión en el sector, transferir los recursos hacia los colectivos más desfavorecidos de la población para reducir la desigualdad existente (p.e.: inversión en nuevos planes y programas, construcción de nuevas infraestructuras, otros).

Figura 4. Distribución del GPT y Slums

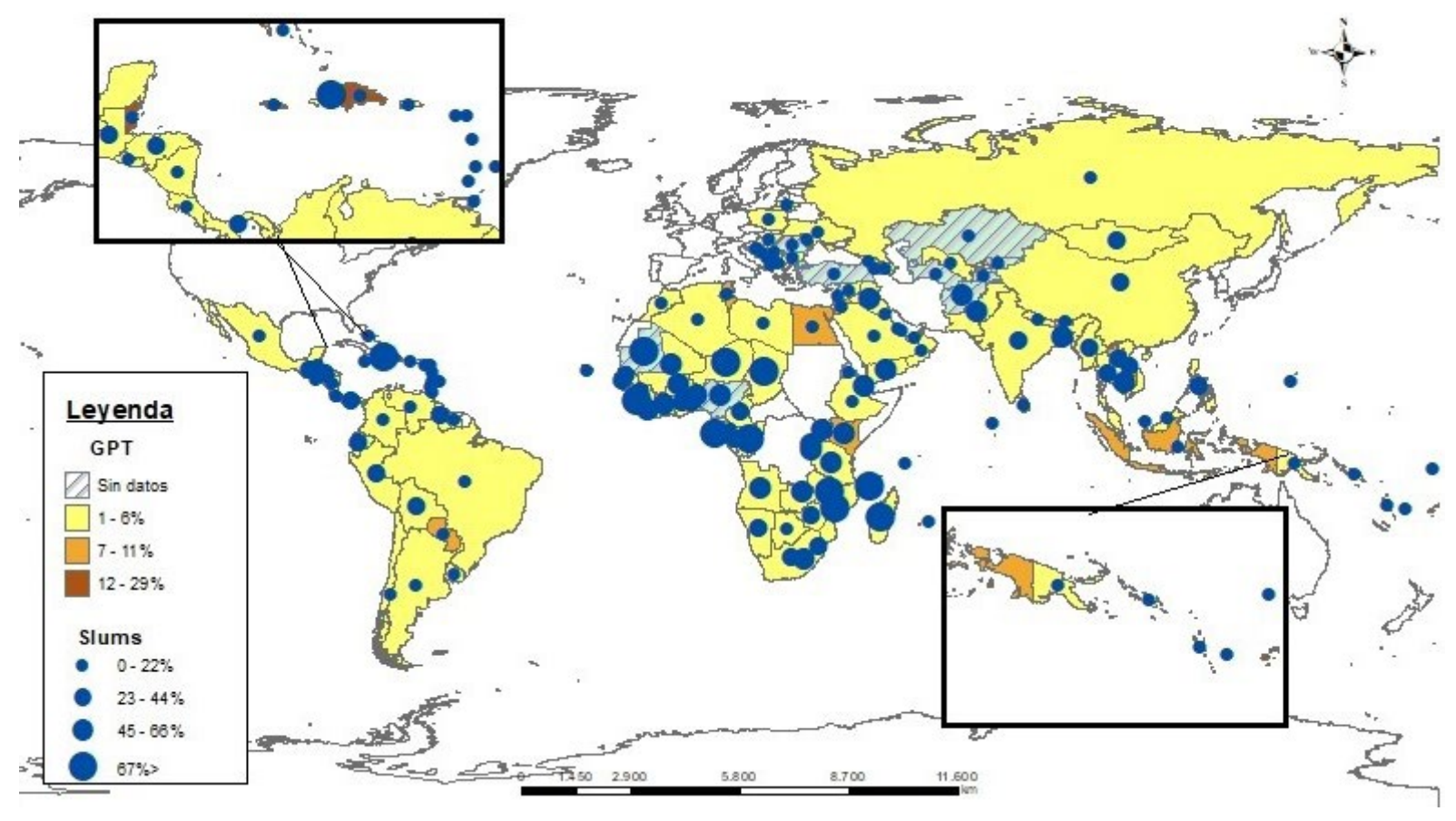

Elaboración propia basada en WTTC (2016) y Banco Mundial (2016c)

Atendiendo a la figura 4, los destinos africanos destacan por una mayor concentración de Slums y escaso GPT, incluyendo aquellos que, pese a ser reconocidos a nivel mundial, se encuentran en esta situación, como Madagascar y Santo Tomé y Príncipe. En América Latina, el GPT es mayor y la existencia de Slums tiende a ser menor, como son Argentina, Costa Rica o Belice. En Asia, vuelven a existir casos tendentes hacia alguna de las dos situaciones descritas para los otros continentes, casos como Indonesia, más similar a la dinámica de los destinos africanos, o Camboya, a la de los destinos latinoamericanos.

\section{La persistencia de la TR y el IP en el destino: grupos en riesgo de exclusión social}

Pese a no haberse hallado ninguna correlación directa con la TR y el IP, estos indicadores presentan altos valores en los destinos objeto de estudio, convirtiéndose en dos condiciones socioeconómicas inherentes para el conjunto de la muestra durante el análisis que aborda el trabajo. 
Tabla 5. Estadísticos descriptivos TR e IP

\begin{tabular}{|c|c|c|c|c|c|}
\hline & N & Mínimo & Máximo & Media & Desviación estándar \\
\hline TR & 152 & 11,05 & 99,23 & 78,4882 & 35,20106 \\
\hline IP & 152 & 0,11 & 77,18 & 45,0311 & 9,38876 \\
\hline N válido (por lista) & 144 & & & & \\
\hline
\end{tabular}

Elaboración propia

Es importante recalcar tanto el alto porcentaje de población que reside con ingresos inferiores a $1,90 \$$ diarios, como los escasos ingresos turísticos netos que contribuyen al desarrollo socioeconómico de los destinos emergentes (tabla 5).

Concretamente, la TR tiende a ser alta en estos destinos, obteniendo una media aritmética del $78,5 \%$ para el conjunto de la muestra. Esto implica que de cada 100 unidades monetarias (u.m.) que percibe el destino por turismo, tan solo 22,5 u.m. permanecerían en la economía para contribuir a su desarrollo socioeconómico, mientras que las 78,5 u.m. restantes revertirían a otras economías. Por su parte, el IP engloba a alrededor del $45 \%$ de la población de estos destinos. Estos datos señalan una tendencia a concentrar los beneficios en los mismos grupos (p. e.: inversión extranjera), alejando a estos destinos del paradigma del alivio de la pobreza a partir del turismo.

La situación descrita conlleva, a su vez, una situación de estratificación turística o de empoderamiento (Boley y McGehee, 2014; Sofield, 2003), a partir de la cual los grupos con mayor participación en los beneficios turísticos dependen de esta actividad para mantener su posición privilegiada, en detrimento de la parte de la población cuyo acceso al beneficio de la actividad turística es nulo, o en su caso, limitado. De este modo, un incremento de las desigualdades económicas supone un empobrecimiento continuado en el tiempo por parte de los grupos más desfavorecidos, independientemente que, en un momento dado, puedan ver incrementados sus ingresos. Estos grupos se acercarían, cada vez más, a la exclusión social.

\section{CONCLUSIONES}

El contraste de hipótesis ha resultado fundamental para estimar la potencialidad del turismo en su contribución a la calidad de vida de la población en los destinos emergentes. Los resultados estadísticos apuntan que el turismo y la pobreza guardan relación:

H1 demuestra que el desarrollo turístico podría contribuir a reducir el porcentaje de personas que residen en las áreas más desfavorecidas o Slums.

H2 recalca que en este desarrollo turístico tendría relevancia el apoyo público y el control que ejerce en el sector, para influir sobre las situaciones de desigualdad existentes entre la población.

H3 demuestra que la gobernanza turística podría influir sobre la calidad de vida de la población más desfavorecida, a partir de los recursos invertidos en el sector. 
Tanto en $\mathrm{H} 2$ como H3, la iniciativa pública juega un papel clave para el desarrollo del destino. Por ello, es necesaria una planificación e implementación de políticas pro-pobres, que se adecuen a las necesidades de los agentes en el destino: iniciativa pública, privada, y comunidad local, e incidan de manera especial sobre los grupos más desfavorecidos y/o en peligro de exclusión social. En este sentido, es oportuno que se identifiquen aquellos aspectos prioritarios sobre los que es más conveniente invertir para beneficiar directamente a estos colectivos, en lugar de al resto de componentes de la cadena de valor.

Dada la significatividad de las tres relaciones analizadas, la actividad turística podría ser concebida como un instrumento para contribuir a que las desigualdades económicas existentes en el destino se incrementen o disminuyan. En este sentido, se prueba que el turismo podría ser utilizado como un elemento estabilizador en la economía del destino. Sin embargo, los elevados índices de IP y TR que concentran los destinos emergentes señalan la necesidad de una reorientación en su desarrollo. Esto supone encauzar el turismo hacia modelos que conlleven una reducción gradual de las desigualdades económicas y el efectivo alivio de la pobreza, a partir de lo que se conoce como el efecto trickle down o "efecto derrame", por el que parte de los beneficios de las clases más altas de la pirámide revierten a la base de la misma, reduciendo estas diferencias. De ello dependerá el papel de la iniciativa pública a la hora de invertir en la actividad turística para controlar que estos recursos no sean acaparados por las "elites de poder", y sean accesibles para los grupos más desfavorecidos en el destino. Solo así se conseguirá una efectiva reducción de la pobreza en su economía.

La iniciativa privada puede convertirse en un importante apoyo en este cometido, al adquirir bienes y servicios locales, contratar trabajadores nacionales, o invertir en formación, promoción y comercialización para dotar de mayor competitividad al destino. Todo ello, actuando bajo principios morales afines a la sostenibilidad turística.

Los resultados de este trabajo esclarecen que el turismo puede ser una vía para el alivio de la pobreza de un país, siempre y cuando exista compromiso por parte de los actores en el destino, llevando hacia un modelo más o menos pro-pobre. De otro modo, este fenómeno puede convertirse en catalizador de subdesarrollo.

En definitiva, es cuestión del propio destino y de sus actores, hacer del turismo la causa de su desarrollo, o, por el contrario, la consecuencia de su subdesarrollo.

Según los últimos estudios empíricos presentados en el Documento de Análisis sobre Turismo Sostenible para el Desarrollo (OMT, 2017b), continúan faltando evidencias acerca de la relación causal entre el turismo y el desarrollo sostenible.

Con la finalización del presente trabajo se pretende estimular deliberaciones en torno a la situación real de los destinos emergentes sobre su desarrollo turístico y su tendencia hacia la sostenibilidad socioeconómica.

Si el turismo es considerado un sector clave para la consecución de los ODS y la Agenda 2030, será necesario que estos países encaucen su desarrollo hacia un modelo que garantice la reducción de la pobreza. De otro modo, el turismo se convertirá en una amenaza para estas economías, y las condiciones de pobreza pueden verse agravadas para la comunidad local. 
Además, se abren nuevas líneas de investigación en relación a la temática del trabajo. Por un lado, conocer y profundizar en las repercusiones que tiene el turismo en los PMA, donde en las últimas décadas esta actividad se ha convertido en uno de los principales motores de exportación para estos países. Por otro, desarrollar e innovar en metodología pro-pobre para estimar y evaluar sus efectos sobre las condiciones de vida de la población desde un enfoque cuantitativo, que ayuden a diseñar modelos turísticos efectivos en la reducción de la pobreza. En este sentido, los modelos econométricos se convierten en una metodología pertinente para abordar, tanto la causalidad de las relaciones de esta tipología de estudios (Croes y Venegas, 2008), como la incidencia de las variables independientes sobre la dependiente, permitiendo realizar un riguroso análisis cuantitativo que permita una posterior interpretación de los resultados, desde un punto de vista cualitativo.

\section{REFERENCIAS BIBLIOGRÁFICAS}

Archer, B., Cooper, C., \& Ruhanen, L. (2005). The positive and negative impacts of tourism. Global tourism, 3, 79-102.

Ariza, M. (2016). Agenda internacional 2030: perspectivas de la Cooperación para el desarroIlo. Revista Internacional de Cooperación y Desarrollo, 3(2), 153-158.

Arshad, M. I., Iqbal, M. A., \& Shahbaz, M. (2018). Pakistan tourism industry and challenges: a review. Asia Pacific Journal of Tourism Research, 23(2), 121-132.

Ashley, C., Boyd, C., \& Goodwin, H. (2000). Pro-poor tourism: putting poverty at the heart of the tourism agenda. Natural Resource perspectives, 51, 1-6.

Banco Mundial (2016). Data. (Consulta: 03/06/2016). https://datos.bancomundial.org/ indicador

Baretje, R. (1982). Tourism's external account and the balance of payments. Annals of Tourism Research, 9 (1), 57-67. doi: 10.1016/0160-7383(82)90034-2

Blake, A. (2008). Tourism and income distribution in East Africa. International Journal of Tourism Research, 10(6), 511-524. doi: 10.1002/jtr.702.

Blázquez-Salom, M. Prólogo en El Turismo en el inicio del Milenio. Editorial Foro de Turismo Responsable: Madrid, 7-9.

Boley, B., \& McGehee, N. (2014). Measuring empowerment: Developing and validating the resident empowerment through tourism scale (RETS). Tourism Management, 45, 85-94. doi: 10.1016/j.tourman.2014.04.003

Bonometti, P., \& Ruiz Seisdedos, S. (2010). La democracia en América Latina y la constante amenaza de la desigualdad. Andamios, 7(13), 11-36.

Britton, S. (1982). La economía política del turismo en el Tercer Mundo. En Jurdao, F. (ed.). Los Mitos del Turismo. Madrid: Endymion, 1992, 301-337.

Browett, J. (1985). The newly industrializing countries and radical theories of development. World Development, 13 (7), 789-803.

Butler, R. (1999). Sustainable tourism: A state-of-the-art review. Tourism geographies, 1(1), 7-25. doi: 10.1080/14616689908721291

Casas A., Soler, A. \& Jaime, V. (2012). El turismo comunitario como instrumento de erradicación de la pobreza: potencialidades para su desarrollo en Cuzco (Perú). Cuadernos de turismo, (30), 91-108. 
CEPAL (2012). Indicadores de pobreza y pobreza extrema utilizadas para el monitoreo de los ODM en América Latina. (Consulta: 10/07/16) http://www.cepal.org/cgi-bin/getprod. asp?xml=/MDG/noticias/paginas/1/40211/P40211.xml\&xsl=/MDG/tpl/p18f.xsl\&base=/MDG/tpl/top-bottom.xsl.

Collier, P., \& Dollar, D. (2001). Can the world cut poverty in half? How policy reform and effective aid can meet international development goals. World Development, 29(11), 1787-1802. doi:10.1596/1813-9450-2403.

Croes, R., \& Vanegas M. (2008). Cointegration and causality between tourism and poverty reduction. Journal of Travel Research, 47(1), 94-103. doi: 10.1177/0047287507312429

Dalton, H. (1920). The measurement of the inequality of incomes. The Economic Journal, 30(119), 348-361.

Davis, J. (2001) Commentary: Tourism research and social theory-expanding the focus. Tourism Geographies, 3, 125-134. doi: 10.1080/14616680010030248

DFID (1999). Tourism and Poverty Elimination: Untapped Potential. Department for International Development London.

Dos Santos, T. (1970) The structure of dependency. American Economic Review, 60 (2), 231-236.

Figueroa-Hernández, E., Mendoza-Ontiveros, M., Pérez-Soto, F., \& Godínez-Montoya, L. (2015). ¿El turismo es una opción para "aliviar" la pobreza? Spanish Journal of Rural Development, 6 (1): 17-28. doi: 10.5261/2015.ESP1.02

Fitzgerald, F. (1981). Sociologies of development. Journal of Contemporary Asia, 11(1), 5-18.

FMI (2012). Ficha técnica: Documentos de estrategia de lucha contra la pobreza (DELP). Washington: (Consulta: 30/08/2016). www.imf.org/external/np/exr/facts/spa/prsps. $\underline{\text { htm }}$

FMI (2016). World Economic Outlook. (Consulta: 05/06/2016). http://www.imf.org/external/ pubs/ft/weo/2016/01/pdf/text.pdf

Frenzel, F., Koens, K., \& Steinbrink, M. (2012). Slum tourism: Poverty, power and ethics (Vol. 32). Routledge. Pp. 21-49.

García, A., \& Lavalle, N. (2012). Algunas consideraciones sobre la actividad turística. Notas en Turismo y Economía, 4, 7-43.

Gutiérrez-Pérez, F.; Medina-Muñoz, D.; \& Medina-Muñoz, R. (2014). Tourism and poverty alleviation: an academic literature review. Tourism \& Management Studies, 10(2), 104-115.

Hall, C. (2010). Power in tourism: Tourism in power. Tourism, power and culture. Anthropological insights, 199-213.

Hanafiah, M., Hemdi, M. \& Ahmad, I. (2016). Does tourism destination competitiveness lead to performance? A case of ASEAN region. Turizam: međunarodni znanstveno-stručni časopis 64 (3), 251-260.

Hardy, A., \& Pearson, L. (2016). Determining Sustainable Tourism in Regions. Sustainability, 8(7), 660. doi:10.3390/su8070660

Harrison, D. (2008). Pro-poor tourism: A critique. Third World Quarterly, 29(5), 851-868. doi: $10.1080 / 01436590802105983$.

Harrison, D. (2015). Development theory and tourism in developing countries: what has theory ever done for us? IJAPS, 11 (1), 53-82. 
Holden, A., Sonne, J. \& Novelli, M. (2011). Tourism and poverty reduction: An interpretation by the poor of Elmina, Ghana. Tourism Planning and Development, 8(3), 317-334. doi: 10.1080/21568316.2011.591160.

Idelhadj, A., Mateos, M., \& García, L. (2012). Turismo responsable, espacios rurales y naturales y cooperación para el desarrollo: a propósito de la Declaración de Tetuán (Marruecos). Pasos: Revista de turismo y patrimonio cultural, 10(5), 651-664.

ITC (2011). Colaboración turística: Desarrollo del turismo en países menos adelantados-Significado económico y prioridades políticas. (Consulta: 12/05/17). www.tradeforum. org/Colaboracion-turistica-Desarrollo-del-turismo-en-paises-menos-adelantados--Significado-economico-y-prioridades-politicas/

Jafari, J. (2005). El turismo como disciplina científica. Política y sociedad, 42(1), 39-56.

Koens, A., \& Wood, R. (2017). An analysis of international employment levels in hospitality occupations using secondary statistics. International Journal of Tourism Research, 19(5), 496-504.

Korstanje, M. (2015): Filosofía del turismo y pobreza: un resumen. Revista DELOS: Desarrollo Local Sostenible, n. 22 (Consulta:20/03/2016). www.eumed.net/rev/delos/22/turismo. html

Lee, T. (2013). Influence analysis of community resident support for sustainable tourism development. Tourism Management, 34, 37-46. doi: 10.1016/i.tourman.2012.03.007

Lejárraga, I., \& Walkenhorst, P. (2007). Diversification by deepening linkages with tourism. Washington D.C., USA: World Bank.

Mowforth, M. \& Munt, I. (1998). Tourism and sustainability: New tourism in the Third World. Londres: Routledge.

Muñoz, F. (2005). La resistible exaltación del turismo. Análisis de un caso paradigmático. Contribuciones a la Economía (Consulta: 22/11/2016). http://www.eumed.net/ ce/2005/fme-06.htm

OMT (2015). Tourism and the Sustainable Development Goals. Organización Mundial del Turismo, Madrid. doi:10.18111/9789284417254

OMT (2016). Conferencia Mundial Sobre Turismo para el Desarrollo. (Consulta 14/01/2016). http://media.unwto.org/es/press-release/2016-05-27/primera-conferencia-mundial-sobre-turismo-para-el-desarrollo-el-primer-mini. Organización Mundial del Turismo, Madrid.

OMT (2017a). World Tourism Highlights 2017 Edition. Organización Mundial de Turismo, Madrid.

OMT (2017b). Documento de Análisis sobre Turismo Sostenible para el Desarrollo. Organización Mundial de Turismo, Madrid.

ONU (1964). Conferencia de las Naciones Unidas sobre Comercio y Desarrollo. Ginebra, Suiza: Organización de las Naciones Unidas, marzo- junio 1964.

ONU (2003). The Challenge of Slums: Global Report on Human Settlements. United Nations Human Settlements Programme.

ONU (2008). Sistema de Cuentas Nacionales 2008. Organización de las Naciones Unidas: Nueva York, EEUU.

ONU (2011). Cuenta Satélite de Turismo: Recomendaciones sobre el Marco Conceptual (TSA RMF 2008). Organización de las Naciones Unidas: Nueva York, EEUU. 
ONU (2016a). Los Objetivos de Desarrollo Sostenible. (Consulta 14/01/2017). www.un.org/ sustainabledevelopment/es/objetivos-de-desarrollo-sostenible/

ONU (2016b). List of Less Developed Countries. (Consulta: 05/06/16). http://www.un.org/en/ development/desa/policy/cdp/ldc/ldc_list.pdf. Organización de las Naciones Unidas: Nueva York, EEUU.

Opperman, M. (1993) Tourism space in developing countries. Annals of Tourism Research, 20 (4), 535-556. Neo-marxist Theories of Development. Beckenham: Croom Helm.

Perroux, F. (1955). Note on the concept of growth poles. In I. Livingstone (ed.) Economic Policy for Development. Harmondsworth: Penguin.

Phillips, A. (1977). The Concept of Development. Review of Political Economy, 8, 7-20.

Pigou, A. (1912). Wealth and welfare. Macmillan and Company, limited.

Puig-Cabrera, M. \& Foronda Robles, C. (2017). Estudio de la fenomenología del turismo a través de la cooperación internacional para el desarrollo. International Journal of World of Tourism, 4 (8), 28-44.

Puppim, J. (2003). Governmental responses to tourism development: three Brazilian case studies. Tourism Management, 24(1), 97-110. doi: 10.1016/S0261-5177(02)00046-8.

Redclift, M. (1987). Sustainable Development: Exploring the Contradictions. London: Routledge.

Sandbrook, C. (2010). Putting leakage in its place: the significance of retained tourism revenue in the local context in rural Uganda. Journal of International Development, 22(1), 124136. doi: $10.1002 /$ jid.1507.

Scheyvens, R. \& Russell, M. (2012). Tourism and Poverty Alleviation in Fiji: Comparing the Impacts of Small- and Large-Scale Tourism Enterprises. Journal of Sustainable Tourism, 20 (3), 417-36. doi: 10.1080/09669582.2011.629049.

Sharpley, R. (2009). Tourism and Sustainable Development: Exploring the Theoretical Divide. Journal of Sustainable Tourism, (8), 1, 1-19. doi:10.1080/09669580008667346.

Sharpley, R., \& Telfer, D. (2014). Tourism and development: concepts and issues. Channel View Publications, pp. 37-60.

Simas, M., Pauliuk, S., Wood, R., Hertwich, E. G., \& Stadler, K. (2017). Correlation between production and consumption-based environmental indicators: The link to affluence and the effect on ranking environmental performance of countries. Ecological Indicators, 76, 317-323.

Sofield, T. H. (2003). Empowerment for sustainable tourism development (Vol. 7). Emerald Group Publishing.

Spenceley, A. and Meyer, D. (2012). Tourism and Poverty Reduction: Theory and Practice in Less Economically Developed Countries. Journal of Sustainable Tourism, 20 (3): 297-317.

Webster, C., \& Ivanov, S. (2014). Transforming competitiveness into economic benefits: Does tourism stimulate economic growth in more competitive destinations? Tourism Management, 40, 137-140. doi: 10.1016/i.tourman.2013.06.003.

Wolf, E. (1982). Europe and the People Without History. Berkeley: University of California Press. WTTC (2016). Data Gateway. (Consulta: 03/06/17). https://tool.wttc.org/. The World Travel \& Tourism Council.

Zhao, W. \& Ritchie, J. (2007). Tourism and poverty alleviation: An integrative research framework. Current Issues in Tourism, 10(2/3), 119-143. doi:10.2167/cit296.0. 
ANEXO I: 152 DESTINOS EMERGENTES (FMI, 2016; OMT; 2017A):

\begin{tabular}{|c|c|c|c|}
\hline - Albania & - Ecuador & - Malaysia & - Serbia \\
\hline - Algeria & - Egypt & - Maldives & - Seychelles \\
\hline - Angola & - El Salvador & - Mali & - Sierra Leone \\
\hline - Antigua and Barbuda & - Eq. Guinea & - Marshall Islands & - Solomon Islands \\
\hline - Argentina & - Eritrea & - Mauritania & - South Africa \\
\hline - Armenia & - Ethiopia & - Mauritius & - South Sudan \\
\hline - Azerbaijan & - Fiji & - Mexico & - Sri Lanka \\
\hline - Bahrain & - FYR Macedonia & - Micronesia & - St. Kitts and Nevis \\
\hline - Bangladesh & - Gabon & - Moldova & - St. Lucia \\
\hline - Barbados & - Georgia & - Mongolia & - St. Vincent- Grenadines \\
\hline - Belarus & - Ghana & - Montenegro & - Sudan \\
\hline - Belize & - Grenada & - Morocco & - Suriname \\
\hline - Benin & - Guatemala & - Mozambique & - Swaziland \\
\hline - Bhutan & - Guinea & - Myanmar & - Syria \\
\hline - Bolivia & - Guinea-Bissau & - Namibia & - Tajikistan \\
\hline - Bosnia-Herzegovina & - Guyana & - Nauru & - Tanzania \\
\hline - Botswana & - Haiti & - Nepal & - Thailand \\
\hline - Brazil & - Honduras & - Nicaragua & - The Bahamas \\
\hline - Brunei Darussalam & - Hungary & - Niger & - The Gambia \\
\hline - Bulgaria & - India & - Nigeria & - Timor-Leste \\
\hline - Burkina Faso & - Indonesia & - Oman & - Togo \\
\hline - Burundi & - Iran & - Pakistan & - Tonga \\
\hline - Cabo Verde & - Iraq & - Palau & - Trinidad and Tobago \\
\hline - Cambodia & - Jamaica & - Panama & - Tunisia \\
\hline - Cameroon & - Jordan & - Papua New Guinea & - Turkey \\
\hline - Cent. Afr. Republic & - Kazakhstan & - Paraguay & - Turkmenistan \\
\hline - Chad & - Kenya & - Peru & - Tuvalu \\
\hline - Chile & - Kiribati & - Philippines & - Uganda \\
\hline - China & - Kosovo & - Poland & - Ukraine \\
\hline - Colombia & - Kuwait & - Qatar & - United Arab Emirates \\
\hline - Comoros & - Kyrgyz Republic & - Republic of Congo & - Uruguay \\
\hline - Costa Rica & - Lao P.D.R. & - Romania & - Uzbekistan \\
\hline - Côte d'Ivoire & - Lebanon & - Russia & - Vanuatu \\
\hline - Croatia & - Lesotho & - Rwanda & - Venezuela \\
\hline - Dem. Rep. Congo & - Liberia & - Samoa & - Vietnam \\
\hline - Djibouti & - Libya & - Sao Tomé and Príncipe & - Yemen \\
\hline - Dominica & - Madagascar & - Saudi Arabia & - Zambia \\
\hline - Dominican Republic & - Malawi & - Senegal & - Zimbabwe \\
\hline
\end{tabular}

